\title{
Downregulation of nucleolar and spindle-associated protein 1 expression suppresses liver cancer cell function
}

\author{
YIFAN WANG ${ }^{1}$, LINLING JU $^{1}$, FENG XIAO ${ }^{2}$, HUI LIU $^{3}$, XI LUO $^{4}$, LIN CHEN $^{1}$, \\ ZHONGHUA LU ${ }^{5}$ and ZHAOLIAN BIAN ${ }^{1}$ \\ ${ }^{1}$ Nantong Institute of Liver Disease, Nantong Third People's Hospital, Nantong University, Nantong, Jiangsu 226006; \\ ${ }^{2}$ Department of Pathology, Nantong Third People's Hospital, Nantong University, Nantong, Jiangsu 226000; \\ ${ }^{3}$ Department of Gerontology, Shanghai Sixth People's Hospital, Shanghai Jiao Tong University, Shanghai 200233; \\ ${ }^{4}$ Department of Clinical Laboratory, Nantong Third People's Hospital, Nantong University, Nantong, Jiangsu 226000; \\ ${ }^{5}$ Department of Liver Disease, Wuxi Fifth People's Hospital, Jiangnan University, Wuxi, Jiangsu 214013, P.R. China
}

Received June 29, 2018; Accepted January 10, 2019

DOI: $10.3892 /$ etm.2019.7314

\begin{abstract}
The aim of the present study was to determine the role of nucleolar and spindle-associated protein 1 (NuSAP1) in human liver cancer. NuSAP1 expression was determined by reverse transcription-quantitative polymerase chain reaction (RT-qPCR), western blotting and immunohistochemistry in hepatocellular carcinoma (HCC) and adjacent tissues. The expression of NuSAP1 gene was detected by RT-qPCR in liver cancer cell lines. Expression information for NuSAP1 was determined using the UALCAN and Oncomine databases. The Kaplan-Meier plotter and The Cancer Genome Atlas databases were used to obtain overall survival data for liver cancer. Liver cancer cell lines HepG2 and Huh-7 were transfected with lentiviral particles to silence the endogenous NuSAP1 gene expression. RT-qPCR and western blotting were performed to confirm the silencing efficiency. Cell Counting Kit-8 was used to estimate the effects of NuSAP1 silencing on HepG2 and Huh-7 cell proliferation. Cell cycle and apoptosis analyses were performed using flow cytometry. Cell invasion was assessed using the Transwell assay with microscopy imaging. The results revealed that the NuSAP1 expression levels in HCC tissues were significantly increased compared with the adjacent tissues. The survival time of patients with HCC with a high NuSAP1 expression was markedly decreased compared with that of patients with HCC
\end{abstract}

Correspondence to: Dr Zhaolian Bian, Nantong Institute of Liver Disease, Nantong Third People's Hospital, Nantong University, 60 Middle Qingnian Road, Nantong, Jiangsu 226006, P.R. China E-mail: bianzhaolian1998@163.com

Dr Zhonghua Lu, Department of Liver Disease, Wuxi Fifth People's Hospital, Jiangnan University, 1,215 Guangrui Road, Wuxi, Jiangsu 214013, P.R. China

E-mail: lu_z_h@126.com

Key words: nucleolar and spindle-associated protein 1, liver cancer, cell proliferation, apoptosis, cell cycle with a low expression level of NuSAP1. Functional studies revealed that NuSAP1 silencing significantly reduced HepG2 and Huh-7 cell proliferation and invasion. Increased apoptosis and cell cycle arrest at the G1 phase were observed following NuSAP1 knockdown. NuSAP1 silencing significantly inhibited the mRNA expression of DNA methyltransferase but not glioma-associated oncogene. NuSAP1 contributed to liver cancer development by reducing apoptosis and promoting cell cycle progression. The abnormal expression level of NuSAP1 may serve a role in promoting liver cancer progression.

\section{Introduction}

Hepatocellular carcinoma (HCC), which accounts for $\sim 80 \%$ of adult primary liver cancer, is the most lethal form of liver cancer worldwide (1), with the prevalence and incidence rates rising worldwide annually (2). Early diagnosis of liver cancer is challenging due to the limited amount of clinical studies and accurate biomarkers (3). In addition, the lack of specific symptoms in the early stages further results in poor prognosis (3).

Nucleolar and spindle-associated protein 1 (NuSAP1) is an important microtubule and chromatin-binding protein. It has been demonstrated to serve a role in microtubule crosslink during mitosis (4-6), regulate the function of kinetochore microtubules (7), and dominate chromosome oscillation (8). Furthermore, NuSAP1 regulates spindle assembly, chromosome segregation and cytokinesis $(4,9)$. Previous studies have reported that NuSAP1 is involved in malignancies, including melanoma $(10,11)$, breast cancer $(12,13)$, glioblastoma $(14)$, HCC (15), prostate cancer (16), glioblastoma multiforme (17), astrocytoma (18) and meningioma (19). Cui et al (20) revealed that NuSAP1 may act as one of the core modules in the suppression of HCC by silymarin. In addition, it has been reported that, during cell division, NuSAP1 acted as a co-factor for the E3 SUMO-protein ligase RanBP2-Ran GTPase-activating protein 1-SUMO-conjugating enzyme UBC9 complex and participated in accurate chromosome segregation (21). The above studies indicated that NuSAP1 serves a role in cancer development. To further understand the role of NuSAP1 in 
liver cancer, the current study investigated the effects of the downregulation of NuSAP1 expression in liver cancer cell lines. Functional studies confirmed the biological significance of the NuSAP1 gene in liver cancer.

\section{Materials and methods}

Patient and tissue samples. Tumorous and adjacent non-tumorous human liver specimens were collected from 47 patients who underwent surgery for HCC at the Nantong Third People's Hospital Affiliated to Nantong University (Nantong, China) between September 2015 and July 2016. All patients in this study were diagnosed with primary HCC. No preoperative radiotherapy or chemotherapy was administered to these patients. Histopathological diagnosis of all patients with HCC was completed by the Department of Pathology. The pathological stage of HCC was determined according to the International Union Against Cancer Tumor-Node-Metastasis (TNM) Classification (22). The baseline characteristics of the participants were summarized in a previous study (23). All tissues used in the current study were frozen immediately following dissection and stored in liquid nitrogen.

Databases. The expression of NuSAP1 in patients with HCC was analyzed using the multiple cancer microarray datasets available from Oncomine (www.oncomine.org) (24). The Cancer Genome Atlas expression information for NuSAP1 was obtained using UALCAN (www.ualcan.path.uab.edu) (25). A 120-month follow-up study was performed, using HCC patient information available from the Kaplan-Meier plotter database (www.kmplot.com) (26), to verify the association between NuSAP1 expression and prognosis in patients with HCC. Furthermore, a 80-month follow-up study was also performed using OncoLnc (www.oncolnc.org) (27).

Cell lines. LO2, HepG2, Huh-7 and SNU-423 cell lines were obtained from the Cell Bank of Type Culture Collection of Chinese Academy of Sciences (Shanghai, China). All cells were cultured in Dulbecco's modified Eagle's medium (DMEM; Thermo Fisher Scientific, Inc., Waltham, MA, USA) supplemented with $10 \%$ fetal bovine serum (FBS; ScienCell Research Laboratories, Inc., San Diego, CA, USA) and 1\% Penicillin-Streptomycin (Thermo Fisher Scientific, Inc.) and maintained at $37^{\circ} \mathrm{C}$ with $50 \mathrm{ml} / 1 \mathrm{CO}_{2}$.

Lentiviral infection. One day prior to infection, the HepG2 or Huh-7 cell suspensions were seeded onto six-well plates, at a density of $1 \times 10^{5}$ cells/well. Two groups included, the siNuSAP1 group, transfected with NuSAP1-siRNA green fluorescent protein (GFP) lentivirus, and the control group, transfected with a scrambled siRNA GFP lentivirus. The following day, DMEM with $10 \% \mathrm{v} / \mathrm{v}$ FBS (complete medium) was removed and replaced with Enhanced Infection Solution (1 ml/well; Shanghai GeneChem Co., Ltd., Shanghai, China), and polybrene (Shanghai GeneChem Co., Ltd.) was diluted to a final concentration of $5 \mu \mathrm{g} / \mathrm{ml}$ with Enhanced Infection Solution. For each cell line, different multiplicity of infection (MOI) values $(20,40,60,80,100$ and 120) were tested, and the final MOI value used in the present study was determined when the efficiency of infection reached $80 \% 3$ days post-infection.
HepG2 cells were infected at a MOI of 120 . Huh-7 cells were infected at a MOI of 60 . After swirling the plate gently to mix the cells, the plate was placed in an incubator with $50 \mathrm{ml} / \mathrm{l}$ $\mathrm{CO}_{2}$ at $37^{\circ} \mathrm{C}$. After $8 \mathrm{~h}$, the medium was removed and replaced by the complete medium. Three days post-transfection, GFP expression was observed in five randomly-selected fields using a fluorescence microscope (magnification, x200). Cells with transfection efficiency $>80 \%$ on day 3 were selected for subsequent analysis. Lentiviruses were purchased from Shanghai GeneChem Co., Ltd. (Shanghai, China). The following target siRNA sequences were used: Scrambled control, 5'-TTCTCCGAACGTGTCACGT-3', siNuSAP4-1, 5'-ATAAGCGTTCACTGACCAA-3', and siNuSAP1 6-1, 5'-CCTTAAAGCTCAAATTCTT-3'.

Reverse transcription-quantitative polymerase chain reaction (RT-qPCR). Total RNA was extracted using TRIzol ${ }^{\circledR}$ reagent (Thermo Fisher Scientific, Inc.) from HCC tumor tissues, adjacent tissues and liver cancer cell lines. The RNA purity and concentration were measured using a NanoDrop ${ }^{\mathrm{TM}} 2000$ spectrophotometer (Thermo Fisher Scientific, Inc.). The Prime Script $^{\mathrm{TM}}$ RT Reagent kit (Takara Biotechnology Co., Ltd., Dalian, China) was used for the RT of RNA, according to the manufacturer's protocol. The reactions were performed using a Bio-Rad Real-Time PCR Detection System (Bio-Rad Laboratories, Inc., Hercules, CA, USA). SYBR ${ }^{\circledR}$ Green Master Mix (Vazyme, Piscataway, NJ, USA) was used for the qPCR. The following primers were used: NuSAP1 forward, 5'-TCA TTTCCTTTTCTTGCCTCA-3' and reverse, 5'-CCCTCA AGTACAGTGACCTGC-3'; glioma-associated oncogene (GLI1) forward, 5'-AACCCTTGGAAGGTGATATGTC-3' and reverse, 5'-TTCATACACAGATTCAGGCTCA-3'; DNA methyltransferase (DNMT1) forward, 5'-CGGCAGACCATC AGGCATTCTAC-3' and reverse, 5'-CACACCTCACAG ACGCCACATC-3'; $\beta$-actin forward, 5'-GGACTTCGAGCA AGAGATGG-3' and reverse, 5'-AGGAAGGAAGGCTGG AAGA-3'. The following thermocycling conditions were used: Initial denaturation at $95^{\circ} \mathrm{C}$ for $5 \mathrm{~min} ; 40$ cycles of $10 \mathrm{sec}$ at $95^{\circ} \mathrm{C}$ and $30 \mathrm{sec}$ at $60^{\circ} \mathrm{C}$. Data were analyzed using the $2^{-\Delta \Delta \mathrm{Cq}}$ method (28).

Western blotting. Tissues and cells were lysed in radioimmunoprecipitation assay buffer (Beyotime Institute of Biotechnology, Shanghai, China). The supernatants of centrifuged lysates $\left(14,000 \mathrm{x} \mathrm{g}\right.$ for $5 \mathrm{~min}$ at $\left.4^{\circ} \mathrm{C}\right)$ were diluted in $5 \mathrm{X}$ Laemmli SDS sample buffer (Beyotime Institute of Biotechnology) at a 4:1 ratio. Total protein was quantified using a bicinchoninic acid assay (Beyotime Institute of Biotechnology) and then boiled at $95^{\circ} \mathrm{C}$ for $5 \mathrm{~min}$. Proteins (35 $\mu \mathrm{g} /$ lane) were separated by SDS-PAGE on $10 \%$ gels. The separated proteins were transferred onto nitrocellulose membranes (Pall Life Sciences, Port Washington, NY, USA), and incubated with primary antibodies against NuSAP1 (cat. no. 12024-I-AP; 1:2,000) and $\beta$-actin (cat. no. HRP-60008; 1:2,000; both ProteinTech Group, Inc., Chicago, IL, USA) overnight at $4^{\circ} \mathrm{C}$. Following washing with tris-buffered saline and Polysorbate 20, the membranes were incubated with horseradish peroxidase-conjugated secondary antibody (cat. no. A00098; 1:2,000; GenScript Co., Ltd., Nanjing, China) for $2 \mathrm{~h}$ at room temperature. The protein-antibody complexes were visualized using the SuperSignal 
West Femto Maximum Sensitivity substrate (Thermo Fisher Scientific, Inc.) and exposed to a medical X-ray film (Denville Scientific Inc., Holliston, MA, USA). Proteins were quantified using the Multi Gauge software (version 3.0; Fujifilm Dimatix Inc, Santa Clara, CA, USA).

Immunohistochemistry $(I H C)$. Tissues were fixed in $10 \%$ formalin for $12 \mathrm{~h}$ at $4^{\circ} \mathrm{C}$ and embedded in paraffin. Paraffin-embedded tissue samples (6- $\mu$ m-thick) were cut. All sections were heated in roaster at $65^{\circ} \mathrm{C}$ for $1 \mathrm{~h}$, and then deparaffinized in dimethylbenzene and rehydrated in a descending alcohol series at room temperature. Antigen retrieval was performed by boiling the tissue sections in citrate buffer (pH 6.0) in a microwave for $30 \mathrm{~min}$. Endogenous peroxidase activity was quenched by treatment with methanol and $3 \%$ hydrogen peroxide for $15 \mathrm{~min}$ at room temperature and background staining was blocked for $1 \mathrm{~h}$ at room temperature with $10 \%$ non-immune goat serum (Thermo Fisher Scientific, Inc.). Tissue sections were incubated with primary antibody against NuSAP1 (cat. no. 12024-I-AP; 1:200; ProteinTech Group, Inc.) overnight at $4^{\circ} \mathrm{C}$. Following washing with $\mathrm{PBS}$, the tissue sections were incubated with a horseradish peroxidase-conjugated secondary antibody (Shanghai Long Island Biotec Co., Ltd, Shanghai, China) for $30 \mathrm{~min}$ at room temperature. Tissue sections were subsequently washed with PBS and stained with 3,3'-diaminobenzidine (DAB; Maixin-Bio, Guangzhou, China) for $60 \mathrm{sec}$. Tissue sections were counterstained with Hematoxylin for $15 \mathrm{sec}$ at room temperature for nuclear staining. Sections without the primary antibody served as negative controls. Histological sections of fixed tissue samples were observed using a light microscope (magnification, x200; Leica Microsystems GmbH, Wetzlar, Germany).

Cell proliferation assay. A total of $100 \mu \mathrm{l}$ of cell suspension (3,000 cells/well) were seeded into each well of a 96-well plate. The plate was subsequently pre-incubated for $12 \mathrm{~h}$ in a humidified incubator at $37^{\circ} \mathrm{C}$ with $5 \% \mathrm{CO}_{2}$. This time point was defined as day 1 . Subsequently, the plate was incubated for $0,24,48$ or $72 \mathrm{~h}$ in the incubator, followed by the addition of $10 \mu \mathrm{l}$ of Cell Counting kit-8 (CCK-8) solution (Dojindo Molecular Technologies, Inc., Kumamoto, Japan) to each well of the plate, and further incubation for $1.5 \mathrm{~h}$. The absorbance was measured at a wavelength of $450 \mathrm{~nm}$ using a microplate reader (Thermo Fisher Scientific, Inc.).

Cell cycle analysis. One day prior to transfection, HepG2 or Huh-7 cells were seeded into six-well plates. Three days post-transfection, cells were washed with PBS, collected and fixed in $70 \%$ ice-cold ethanol and directly stored in $70 \%$ ethanol overnight at $-20^{\circ} \mathrm{C}$. Prior to analysis, the cells were incubated in $500 \mu \mathrm{l}$ sample buffer [50 $\mu \mathrm{g} / \mathrm{ml}$ propidium iodide (Merck KGaA, Darmstadt, Germany) and $0.25 \mathrm{mg} / \mathrm{ml}$ of RNase A (Takara Bio, Inc., Otsu, Japan)] for $30 \mathrm{~min}$ at room temperature. The proportion of cells in each phase was detected using a BD FACSCalibur Flow Cytometer (BD Biosciences, San Jose, CA, USA) and analyzed using FlowJo software (FlowJo, LLC, Ashland, OR, USA).

Apoptosis analysis. One day prior to the transfection, HepG2 or Huh-7 cells were seeded into six-well plates.
Three days post-transfection, cells were harvested and washed with PBS. Cell apoptosis was assessed using the Annexin V-Allophycocyanin Apoptosis Detection kit I (BD Biosciences). Cells were centrifuged (200 x g for $5 \mathrm{~min}$ at room temperature) and resuspended in $500 \mu \mathrm{l}$ of binding buffer to the density of $1 \times 10^{6}$ cells $/ \mathrm{ml}$. Subsequently, $100-\mu 1$ cell suspension was incubated with $5 \mu 1$ 7-aminoactinomycin $\mathrm{D}$ and $5 \mu \mathrm{l}$ Annexin V-fluorescein isothiocyanate for $15 \mathrm{~min}$ at room temperature, in the dark. Apoptotic cells were detected using a BD FACSCalibur Flow cytometry (BD Biosciences) and analyzed using FlowJo software.

Transwell invasion assay. Transwell filters were coated with Matrigel $(3.9 \mu \mathrm{g} / \mu \mathrm{l} ; 60-80 \mu \mathrm{l})$ on the upper surface of the polycarbonic membrane (diameter, $6.5 \mathrm{~mm}$; pore size, $8 \mu \mathrm{m}$ ). The Matrigel solidified following incubation at $37^{\circ} \mathrm{C}$ for $6 \mathrm{~h}$. Cells $\left(1 \times 10^{5}\right)$ suspended in $200 \mu \mathrm{l}$ serum-free DMEM were added into the upper compartment of the chamber. Simultaneously, $600 \mu \mathrm{l}$ complete medium was added into the bottom compartment of the chamber. The medium was removed from the upper chamber following incubation for $48 \mathrm{~h}$ at $37^{\circ} \mathrm{C}$ with $5 \% \mathrm{CO}_{2}$. The Matrigel was cleaned with a cotton swab. The cells migrated from the Matrigel into the pores of the filter were fixed with $100 \%$ methanol, stained with crystal violet (Beyotime Institute of Biotechnology) for $30 \mathrm{~min}$ at room temperature, washed with PBS and dried at room temperature. The cells invading through the Matrigel were observed using an inverted microscope (magnification, x200). Each assay was repeated three times.

Statistical analysis. GraphPad Prism was used for data analysis (version 6.0; GraphPad Software, Inc., La Jolla, CA, USA). Student's t-test and two-way analysis of variance followed by Tukey's post hoc test were used in the current study. Significance of NuSAP1 expression in association with clinicopathological parameters was calculated using the Chi-square test. A paired t-test was used to analyze the differences of NuSAP1 expression levels between HCC tissues and corresponding adjacent normal tissues. $\mathrm{P}<0.05$ was considered to indicate a statistically significant difference. All data are presented as the mean \pm standard error of the mean. All the experiments were repeated at least three times.

\section{Results}

NuSAP1 is overexpressed in HCC tissues and HepG2 and Huh-7 cell lines. The expression of NuSAP1 in HCC was analyzed using the multiple cancer microarray datasets available from Oncomine and it was determined that the mRNA expression levels of NuSAP1 in HCC tissues were markedly increased compared with those in the normal tissues (Fig. 1A). The Cancer Genome Atlas expression information for NuSAP1 was obtained using UALCAN (Fig. 1B). To improve the understanding of the clinical relevance of NuSAP1 expression in HCC, the association between NuSAP1 expression and clinicopathological parameters was examined (Table I). According to the median value of relative NuSAP1 mRNA expression in HCC tissues, 47 HCC patients were subsequently divided into NuSAP1-high $(n=24)$ and NuSAP1-low groups $(n=23)$. The statistical analysis revealed that a high level of NuSAP1 
A
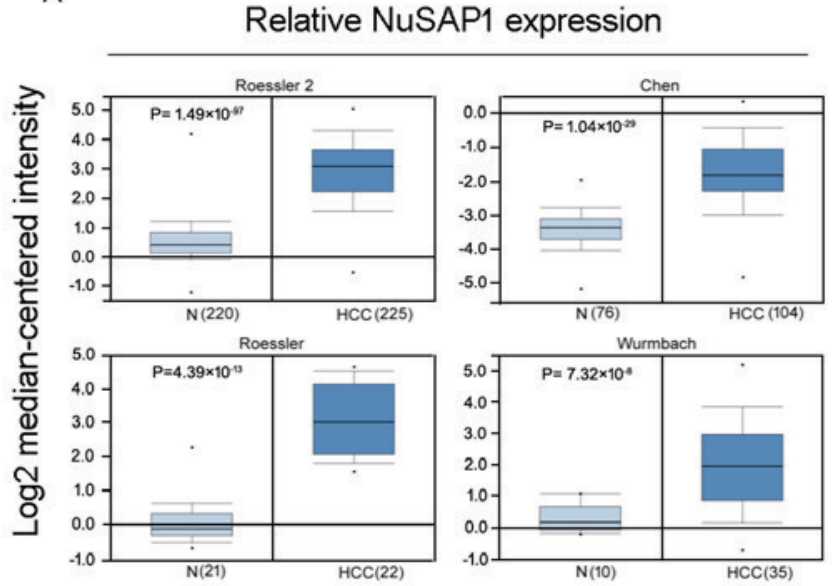

C

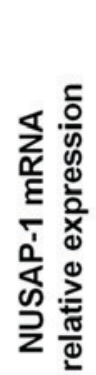

E

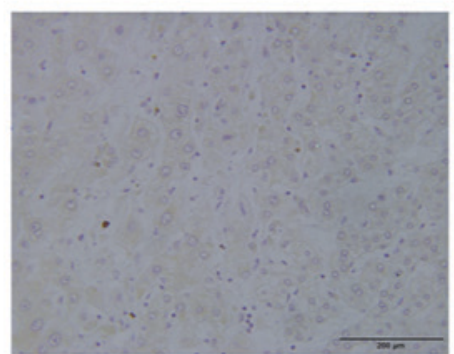

Adjacent tissue

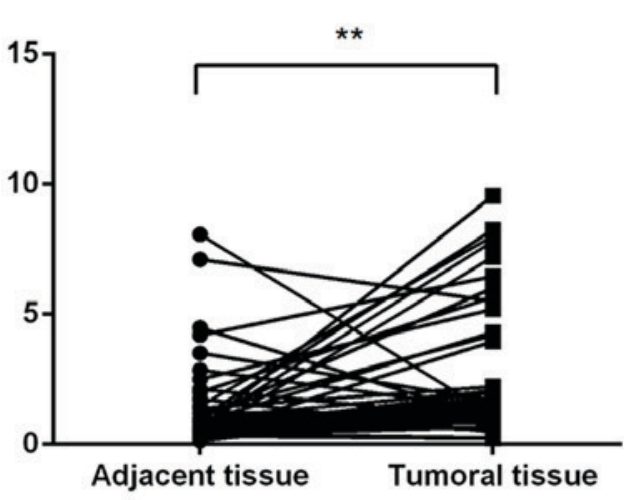

B

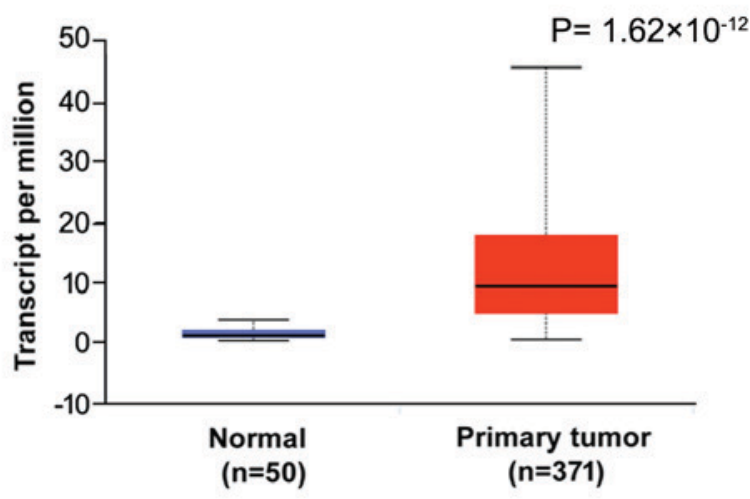

D
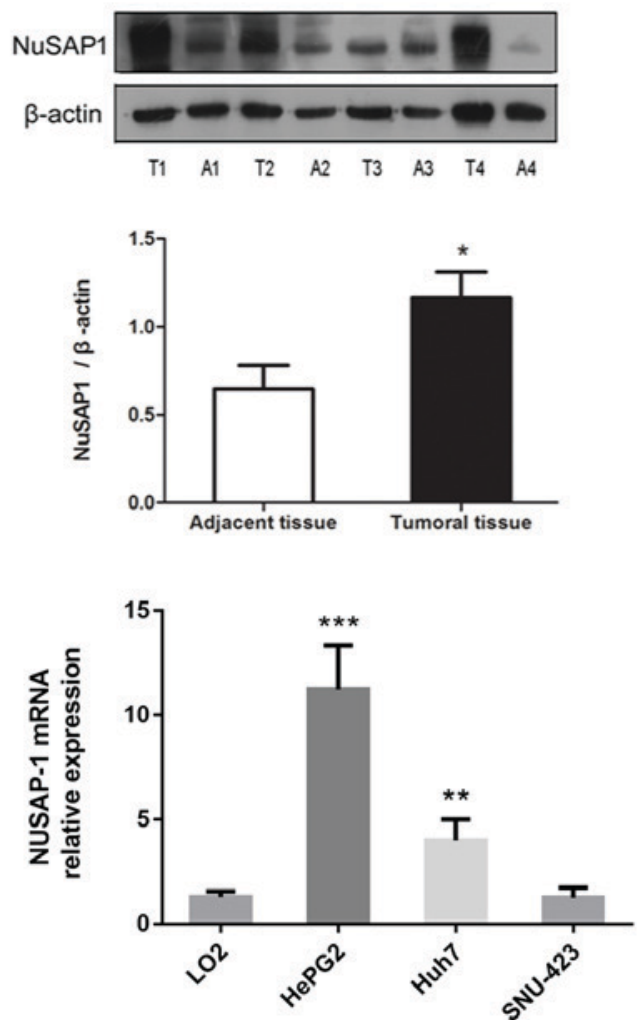

Figure 1. NuSAP1 is overexpressed in HCC tissues and HepG2 and Huh-7 cell lines. (A) NuSAP1 is overexpressed in human HCC tissues, as compared with that in normal tissues, in multiple cancer microarray datasets available from Oncomine. (B) NuSAP1 is overexpressed in human HCC tissues compared with normal tissues, in datasets available from The Cancer Genome Atlas. (C) mRNA expression levels of NuSAP1 in HCC (n=47) and paired adjacent tissue samples $(\mathrm{n}=47)$ were determined by reverse transcription-quantitative polymerase chain reaction. (D) Protein expression levels of NuSAP1 in HCC ( $\mathrm{n}=47$ ) and paired adjacent tissue samples $(\mathrm{n}=47)$ were determined by western blotting. Gray values of the western blot were quantified. Data were normalized to $\beta$-actin. (E) Expression level of NuSAP1 in HCC and paired adjacent tissues determined by immunohistochemistry. Scale bar, $200 \mu \mathrm{m}$. (F) Relative NuSAP1 mRNA levels in LO2, HepG2, Huh-7 and SNU-423 cell lines were determined by reverse transcription-quantitative polymerase chain reaction. $\beta$-actin was used as the internal control. ${ }^{*} \mathrm{P}<0.05$ vs. with adjacent tissue. ${ }^{* *} \mathrm{P}<0.01$ and ${ }^{* * *} \mathrm{P}<0.001$ vs. LO2 cell line. HCC, hepatocellular carcinoma; NuSAP1, nucleolar and spindle-associated protein 1; T, tumor tissue; A, adjacent tissue.

expression in HCC was significantly associated with the TNM stage $(\mathrm{P}=0.011)$ and lymph node metastasis $(\mathrm{P}=0.019)$. However, NuSAP1 expression was not associated with the other clinicopathological features, including age $(\mathrm{P}=0.77)$, sex $(P=0.371)$, serum level of $\alpha$-fetoprotein $(P=0.494)$, hepatitis $\mathrm{B}$ virus infection $(\mathrm{P}=0.286)$ and tumor size $(\mathrm{P}=0.248)$. The current data indicate that elevated NuSAP1 expression may serve a role in HCC progression, and may be a valuable biomarker for this disease. To investigate whether the NuSAP1 expression was altered in HCC tissues, RT-qPCR and western blotting were used to detect the NuSAP1 levels in 47 paired tumor and adjacent tissues. The mRNA and protein levels of NuSAP1 in the tumor tissues were significantly increased compared with the adjacent tissues (Fig. 1C and D). Furthermore, the expression level of NuSAP1 was detected by IHC in 47 paired tumor and adjacent tissues (Fig. 1E). The results revealed that the expression of NuSAP1 was increased in HCC tissues compared with adjacent tissues. To reveal the potential role of NuSAP1 in HCC, NuSAP1 expression was examined in four liver cancer cell lines: HepG2, Huh-7 and 
Table I. Association between NuSAP1 expression and the clinicopathological characteristics of the 47 patients with HCC.

\begin{tabular}{|c|c|c|c|c|}
\hline \multirow[b]{2}{*}{ Characteristics } & \multirow[b]{2}{*}{ Total, n } & \multicolumn{2}{|c|}{ NuSAP1 expression } & \multirow[b]{2}{*}{ P-value } \\
\hline & & Low, $n=23$ & High, n=24 & \\
\hline \multicolumn{5}{|l|}{ Age, years } \\
\hline$\leq 56$ & 28 & 13 & 15 & 0.77 \\
\hline$>56$ & 19 & 10 & 9 & \\
\hline \multicolumn{5}{|l|}{ Gender } \\
\hline Female & 18 & 7 & 11 & 0.371 \\
\hline Male & 29 & 16 & 13 & \\
\hline \multicolumn{5}{|c|}{ Serum AFP, ng/ml } \\
\hline$\leq 400$ & 36 & 19 & 17 & 0.494 \\
\hline$>400$ & 11 & 4 & 7 & \\
\hline \multicolumn{5}{|l|}{ HBV infection } \\
\hline Positive & 38 & 17 & 21 & 0.286 \\
\hline Negative & 9 & 6 & 3 & \\
\hline \multicolumn{5}{|c|}{ TNM stage, I/II/III } \\
\hline $\mathrm{I}+\mathrm{II}$ & 32 & 20 & 12 & 0.011 \\
\hline III & 15 & 3 & 12 & \\
\hline \multicolumn{5}{|l|}{ Tumor size, $\mathrm{cm}$} \\
\hline$\leq 5$ & 24 & 14 & 10 & 0.248 \\
\hline$>5$ & 23 & 9 & 14 & \\
\hline \multicolumn{5}{|c|}{ Lymph node metastasis } \\
\hline Positive & 20 & 14 & 6 & 0.019 \\
\hline Negative & 27 & 9 & 18 & \\
\hline
\end{tabular}

HCC, hepatocellular carcinoma; AFP, $\alpha$-fetoprotein; HBV, hepatitis B virus; TNM, tumor, node, metastasis .

SNU-423 as well as the normal human hepatic cell line LO2 (Fig. 1F). The expression of NuSAP1 was significantly higher in HepG2 and Huh-7 cells compared with LO2 cells. There were no significant changes in NuSAP1 expression level in the SNU-423 cell line compared with LO2. Therefore, the HepG2 and Huh-7 cell lines were selected for future investigation.

High NuSAPl expression is associated with poor prognosis in HCC. To verify the association between NuSAP1 expression and prognosis in HCC, a 120-month follow-up study was performed using HCC patient information available from the Kaplan-Meier plotter database (Fig. 2A) and the result was consistent with OncoLnc (Fig. 2B). The association between the NuSAP1 expression level and prognosis was analyzed. Patients with a higher NuSAP1 expression level exhibited a markedly shorter overall survival compared with those with a lower NuSAP1 expression level.

NuSAP1 silencing inhibits the proliferation and invasion of HepG2 and Huh7 cells. Following siRNA lentiviral transfection, RT-qPCR was performed to determine the expression levels of NuSAP1 in different groups. The results revealed that NuSAP1-siRNA (siRNA 4-1 and siRNA 6-1) decreased the expression of endogenous NuSAP1 mRNA in HepG2 cells (Fig. 3A). Simultaneously, the protein expression of NuSAP1 in NuSAP1-siRNA-treated cells was suppressed compared with cells transfected with the scrambled control (Fig. 3B and C). The results revealed that siRNA 6-1 exhibited a higher efficiency compared with siRNA 4-1. Therefore, siRNA 6-1 was selected for further investigation. In order to determine whether NuSAP1 exerted a tumor facilitating function, the expression of NuSAP1 was suppressed through a lentiviral vector system using siRNA 6-1 in HepG2 and Huh-7 cells with high endogenous NuSAP1 levels. The NuSAP1 expression levels were determined by RT-qPCR and western blotting. To investigate whether NuSAP1 served a role in cell growth in HepG2 and Huh-7 cells, cell proliferation was detected using the CCK-8, and invasive potential was determined by the Transwell assay. The results revealed that the downregulation of NuSAP1 markedly suppressed cell proliferation (Fig. 4A and B) and the invasive capability (Fig. 4C and D) of both HepG2 and Huh-7 cells.

NuSAPl silencing induces cell cycle progression and cell apoptosis. To investigate the growth-suppressing effect of NuSAP1-siRNA on HepG2 and Huh-7 cells, the cell cycle distribution was analyzed by flow cytometry. NuSAP1-siRNA significantly increased the fraction of G1-phase cells in both HepG2 and Huh-7 cells compared with the control group, and decreased the proportion of S-phase cells in the NuSAP1-siRNA group in Huh7 cells (Fig. 5A and B). The results indicated that NuSAP1 silencing may induce cell cycle 

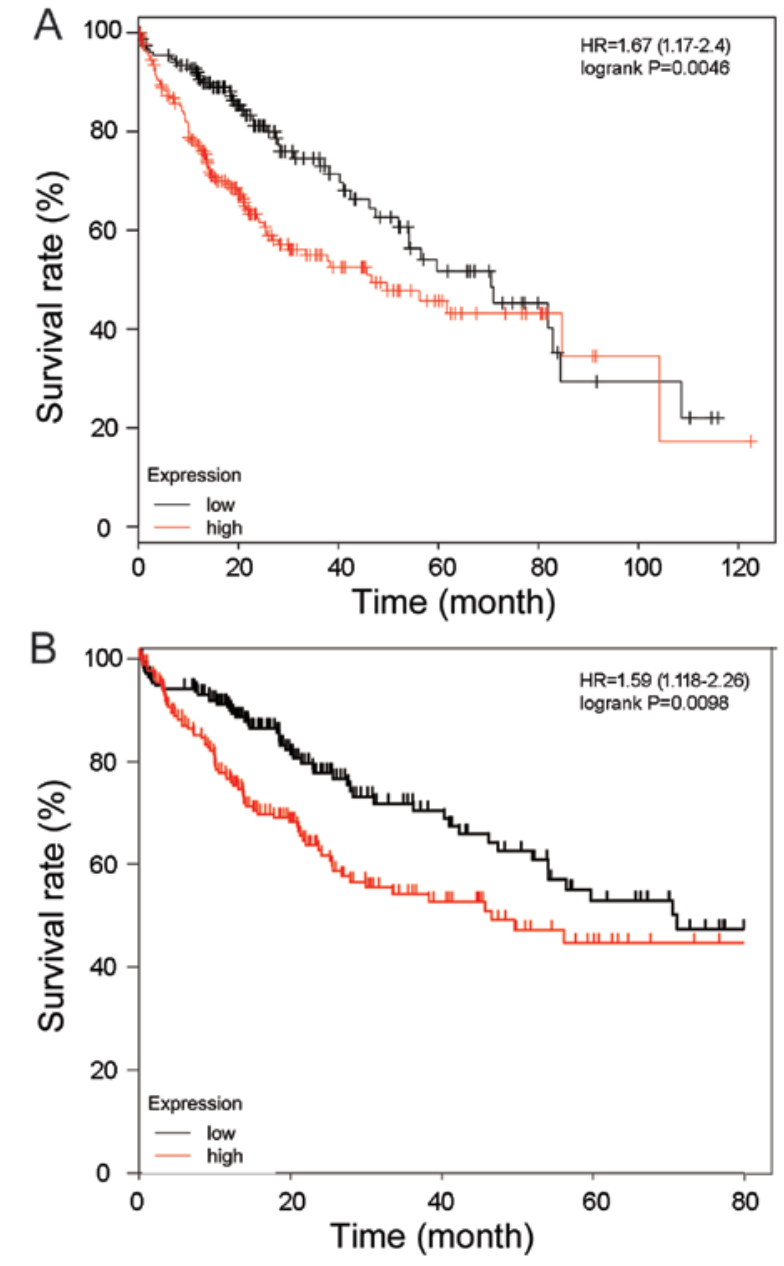

Figure 2. Association between the expression level of nucleolar and spindle-associated protein 1 and prognosis of patients with hepatocellular carcinoma. (A) The difference in survival between the high-and low-level groups was analyzed by the log-rank test from the Kaplan-Meier plotter database $(\mathrm{P}=0.0046)$. (B) The difference in survival between the high-and low-level groups was analyzed by the log-rank test from The Cancer Genome Atlas database $(\mathrm{P}=0.0098)$.

arrest at the G1 phase. The current study further investigated whether NuSAP1 silencing was associated with apoptosis in HepG2 and Huh-7 cells. The number of cells with NuSAP1 gene silencing that exhibited apoptosis was significantly higher compared with the control group (Fig. 5C and D). Lentiviral transfection exhibited considerable infection efficiency toward cells, however it has intrinsic cell toxicity (data not shown). The MOI value used in the current experiment resulted in the most efficient knockdown ratio of NuSAP1 at both mRNA and protein levels (data not shown). Equal MOI was used for the scrambled control group and, therefore, the conclusion was not affected. The above data suggested that the silencing of NuSAP1 interrupted cell cycle progression and affected cell survival.

NuSAP1 silencing inhibits the DNMT1 but not the GLI 1 gene expression in liver cancer cells. A prioritized set of 841 enriched GLI1-binding regions was previously defined by Peterson et al, (29), which included NuSAP1. GLI1 is involved in the intracellular signal transduction controlled by the Hedgehog family of secreted molecules (30). The current study
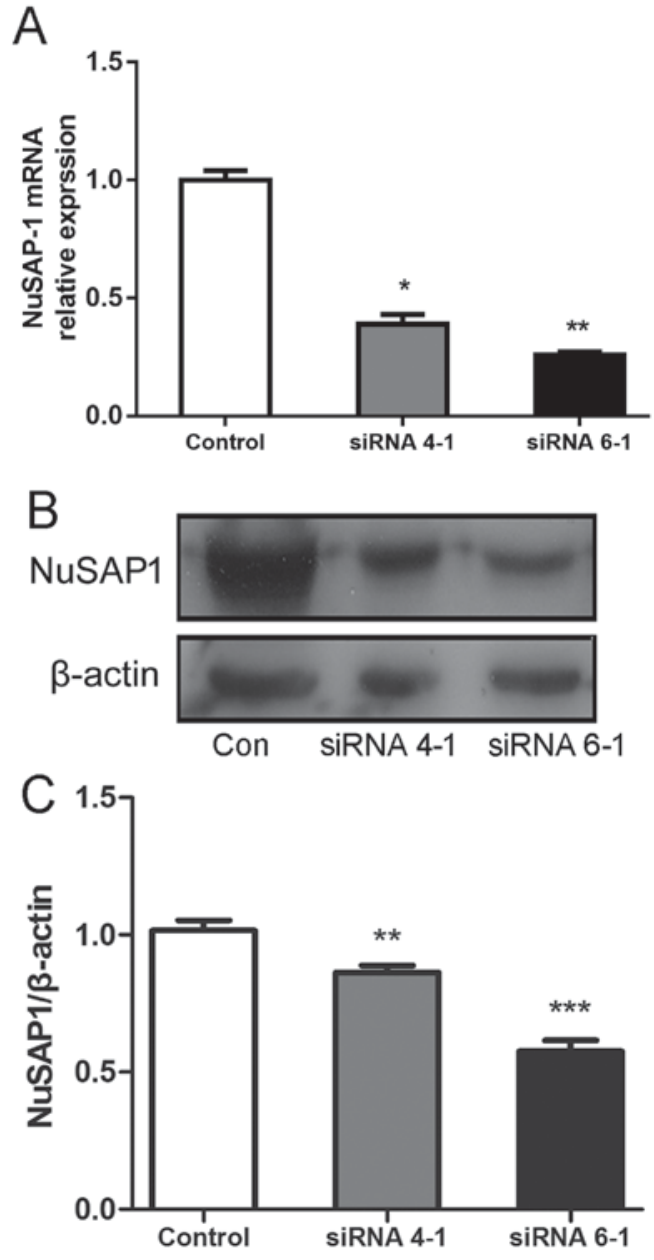

Figure 3. Downregulation of NuSAP1 by lentiviral infection. (A) A total of $72 \mathrm{~h}$ after lentiviral transfection (siRNA 4-1 and siRNA 6-1), the relative NuSAP1 mRNA expression was significantly inhibited in HepG2 NuSAP1-siRNA-silenced cells compared with HepG2 negative control cells, as determined by reverse transcription-quantitative polymerase chain reaction. (B) Western blotting of NuSAP1-depletion efficiency in HepG2 cells. (C) Gray value analysis of western blotting. Data were normalized to $\beta$-actin, which was used as the internal control. ${ }^{*} \mathrm{P}<0.05,{ }^{* *} \mathrm{P}<0.01$ and ${ }^{* * * *} \mathrm{P}<0.001$ vs. the control. NuSAP1, nucleolar and spindle-associated protein 1; siRNA, small interfering RNA.

further investigated whether the Hedgehog (HH)/Gli pathway was associated with NuSAP1. The results revealed no marked alteration in GLI1 mRNA expression levels following NuSAP1 knockdown in the HepG2 cell line (Fig. 6A), which suggested that NuSAP1 may not regulate the biological function of liver cancer through the HH/Gli pathway. NuSAP1 influences the DNA damage response by controlling breast cancer type 1 susceptibility protein (BRCA1) expression levels, and global DNA methylation is affected by BRCA1 through the regulation of DNMT1 $(31,32)$. Thus, the expression of DNMT1 was also detected by RT-qPCR. The results revealed that NuSAP1 silencing significantly inhibited the mRNA level of DNMT1 in HepG2 cells compared with the control group (Fig. 6B).

\section{Discussion}

NuSAP1 is a microtubule and chromatin-binding protein that serves a role in spindle assembly (33). When NuSAP1 is overexpressed, microtubules aggregate into bundles $(4,6)$. 
A

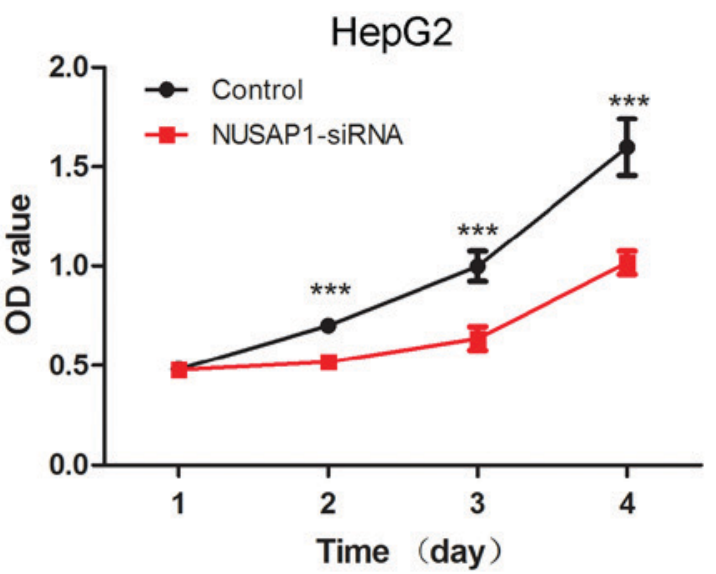

$\mathrm{B}$

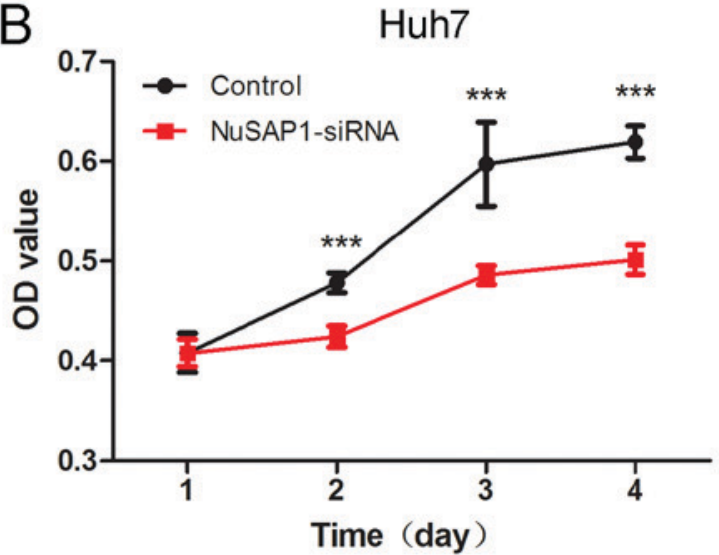

C

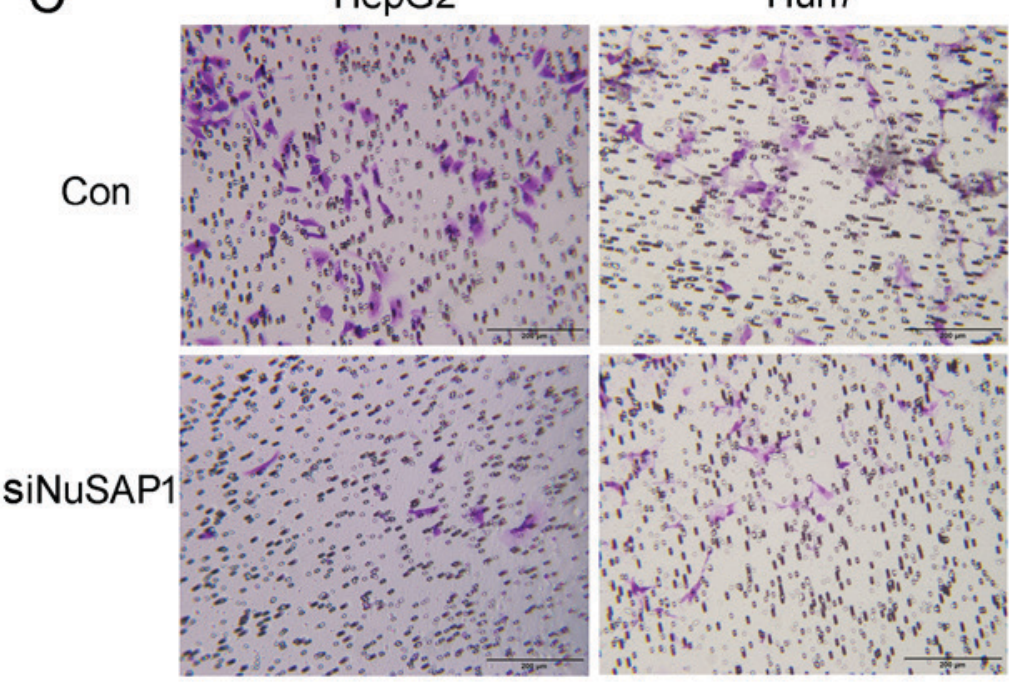

D

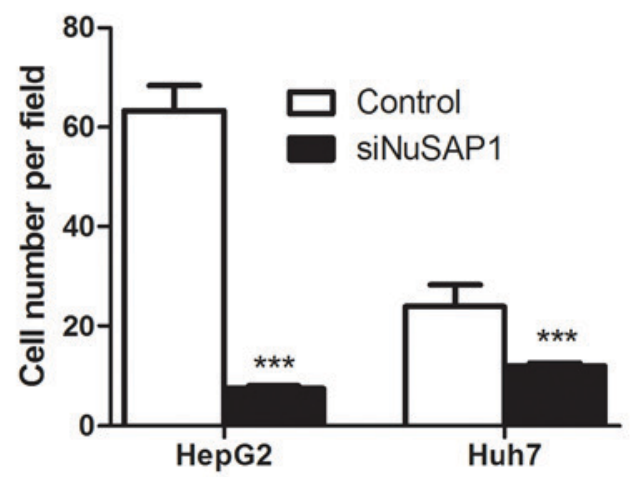

Figure 4. Effects of NuSAP1 silencing on HepG2 and Huh-7 cell proliferation and invasion. The effect of NuSAP1 knockdown on (A) HepG2 and (B) Huh-7 cell proliferation was evaluated by the Cell Counting Kit-8 assay, indicating that NuSAP1 knockdown inhibited cell growth after siNuSAP1 transfection for $72 \mathrm{~h}$. (C) Representative micrographs of the Transwell invasion assay in HepG2 and Huh-7 cells transfected with siNuSAP1. Scale bar, $200 \mu \mathrm{m}$. (D) Knockdown of NuSAP1 suppressed the HepG2 and Huh-7 cell invasive potential compared with the control group. The error bar indicates the SEM. ${ }^{* * *} \mathrm{P}<0.001$ vs. the control group. NuSAP1, nucleolar and spindle-associated protein 1.

NuSAP1 deficiency in mice causes early embryonic lethality (34). The upregulation of NuSAP1 has been reported in a number of different human malignancies; however, few studies have demonstrated the altered expression of NuSAP1 in liver cancer $(10-14,16,19)$. Therefore, to clarify the roles of NuSAP1 in liver cancer, the expression and biological functions of this gene were investigated. RT-qPCR and western blotting detected NuSAP1 protein expression in HCC tumor and adjacent tissues. The results revealed that NuSAP1 was upregulated in HCC tumor tissues, which was in accordance with a previous study (15). This result indicated that NuSAP1 is closely associated with HCC and may be used as a biomarker. Subsequently, the expression of NuSAP1 was determined in different HCC cell lines by RT-qPCR. The results revealed that NuSAP1 was highly expressed in HepG2 and Huh-7 cell lines. Although there was no significant alteration in NuSAP1 expression levels in SNU-423, compared with the normal human hepatic cell line LO2, SNU-423 may be a suitable cell line for overexpression of NuSAP1 to validate the function of this gene in future studies.
After years of optimization and improvements, lentiviral packaging is currently a mature genetic engineering technology applied in numerous studies $(35,36)$. In the present study, NuSAP1 gene expression was effectively suppressed in the HepG2 and Huh-7 cell lines by lentivirus-mediated siRNA. Proliferation is not only necessary for the development and tissue homeostasis during adult life, it is also a hallmark of malignancy (37). To identify genes associated with the clinical outcome of breast cancer, NuSAP1 was detected in four independent studies (38). Therefore, in addition to being a reliable proliferation marker, NuSAP1 is a diagnostic marker for breast cancer and, potentially, other tumor types (38). The current data revealed that cell proliferation and invasion were significantly decreased following silencing of NuSAP1, indicating that its overexpression may enhance cell activity.

Cell cycle assays were performed to investigate the mechanism through which NuSAP1 promotes cell proliferation. The results revealed that the decrease in NuSAP1 expression in liver cancer cells delayed cell cycle progression by arresting the cycle at the G1 phase. It may be hypothesized that an 
A

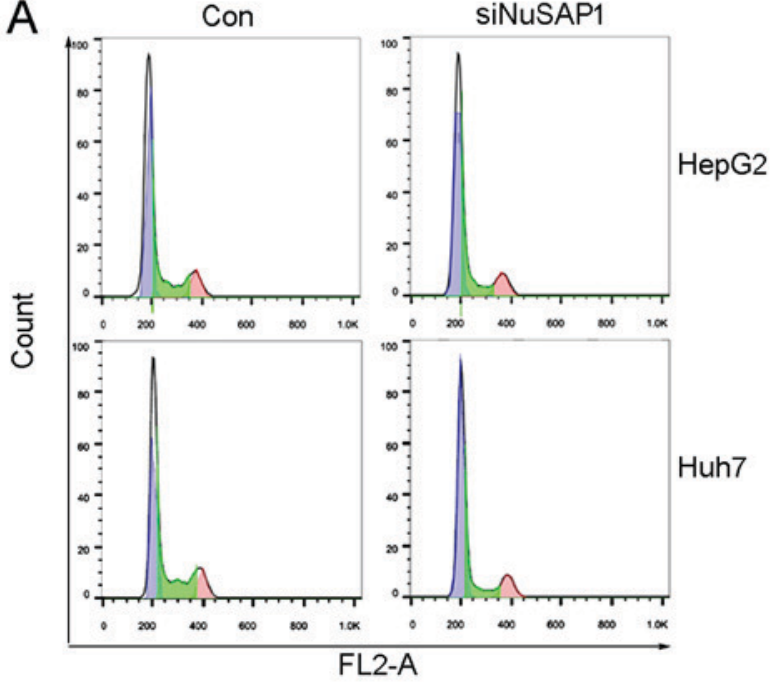

C

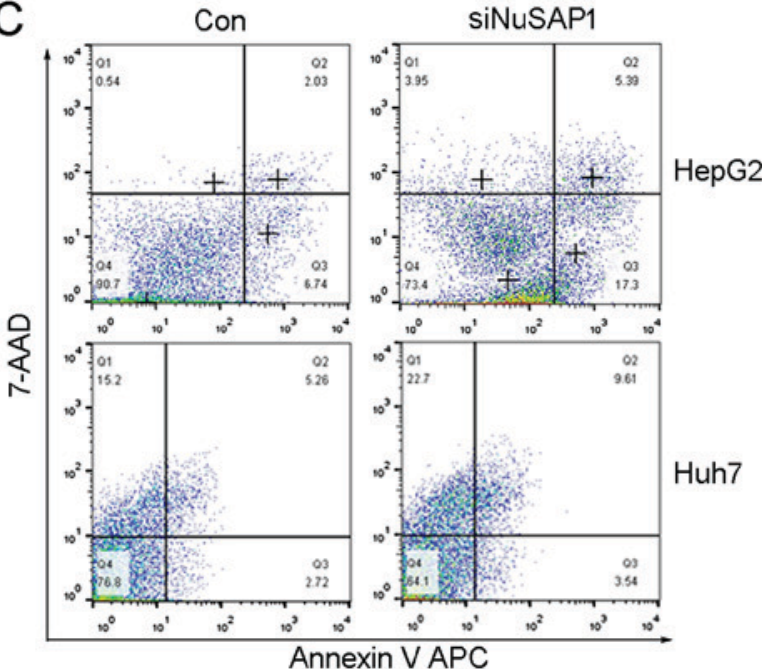

B
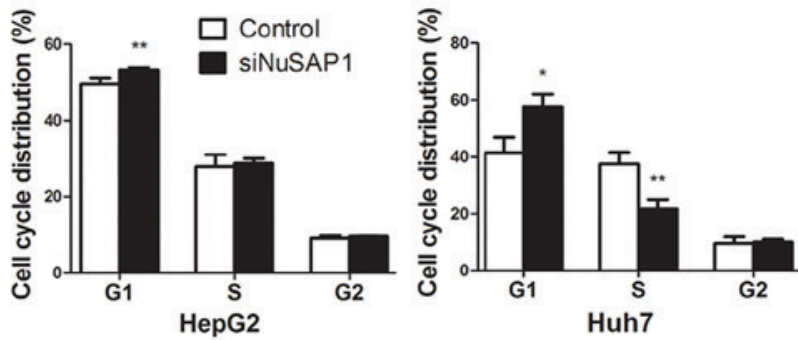

D

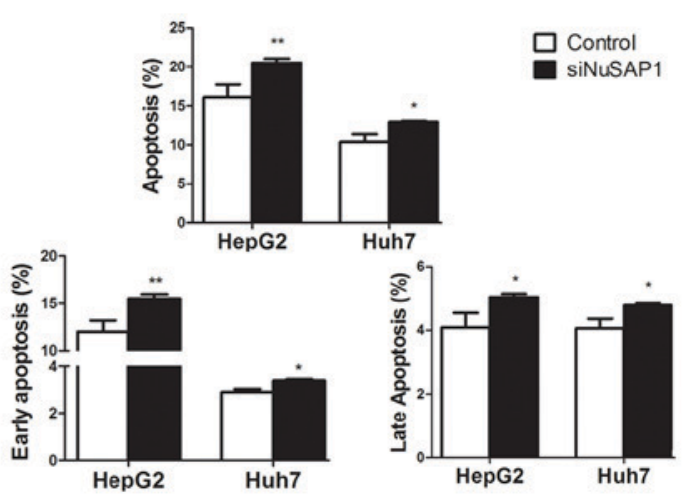

Figure 5. Effects of NuSAP1 silencing on HepG2 and Huh-7 cell cycle distribution and apoptosis. (A) Flow cytometric analysis of the cell cycle distribution in HepG2 and Huh-7 cells treated with siNuSAP1. (B) NuSAP1 knockdown increased cell cycle arrest at the G1-phase compared with the control group. (C) Flow cytometric analysis of the apoptotic rate in HepG2 and Huh-7 cells following siNuSAP1 treatment for $72 \mathrm{~h}$. (D) NuSAP1 knockdown increased cell apoptosis compared with the control group. ${ }^{*} \mathrm{P}<0.05$ and ${ }^{* *} \mathrm{P}<0.01$ vs. with the control. NuSAP1, nucleolar and spindle-associated protein 1 ; si, small interfering RNA; APC, allophycocyanin; 7-AAD, 7-aminoactinomycin D.

upregulated NuSAP1 expression induces a dysregulated cell cycle, which leads to the abnormal proliferation of tumor cells. Therefore, the accelerated liver cancer cell proliferation may be inhibited due to the decreased expression of NuSAP1.

A markedly increased apoptosis in NuSAP1-silenced liver cancer cells was observed in the current study. The mechanisms of apoptosis caused by the knockdown of NuSAP1 remain to be elucidated. The present results indicated that NuSAP1 serves a role in maintaining liver cancer cell growth. These data strongly suggest that NuSAP1 may affects tumor cell proliferation and apoptosis, which contributes to the pathogenesis of liver cancer.

A previous study revealed that NUSAP1 promotes invasion, migration and metastasis by modulating family with sequence similarity 101 member B, a transforming growth factor b1 signaling effector involved in the epithelial to mesenchymal transition (39). Another study indicated a strong correlation between E2F1 and NuSAP expression in human prostate cancer samples (16). In the present study, to further investigate the underlying molecular mechanism of NuSAP1 in liver cancer, the GLI1 and DNMT1 expression levels were measured in liver cancer cells using RT-qPCR. It has been previously reported that NuSAP1 promoted the aggressiveness of astrocytoma by activating the $\mathrm{HH}$ signaling pathway (18). However, no significant difference was observed in the GLI1 gene expression level following NuSAP1 silencing in the current study. A previous study revealed that NuSAP1 influenced the DNA damage response by controlling breast cancer type 1 susceptibility protein (BRCA1) expression levels (31). Global DNA methylation is affected by BRCA1 through the regulation of DNMT1 (32). Therefore, determining whether NuSAP1 may regulate the DNMT1 expression would be noteworthy. The present results indicated that NuSAP1 silencing inhibited the expression of the DNMT1 gene in liver cancer cells. However, the underlying mechanism remains unclear and requires further research. Overall, these results suggested that NuSAP1 silencing may have no effect on the $\mathrm{HH}$ signaling pathway. 
A

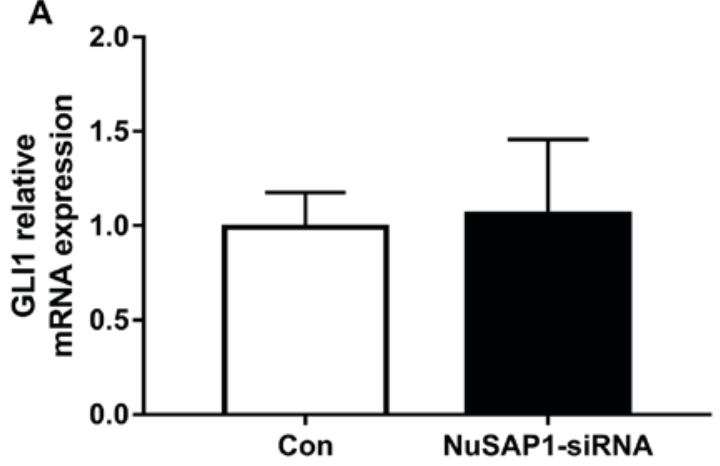

8

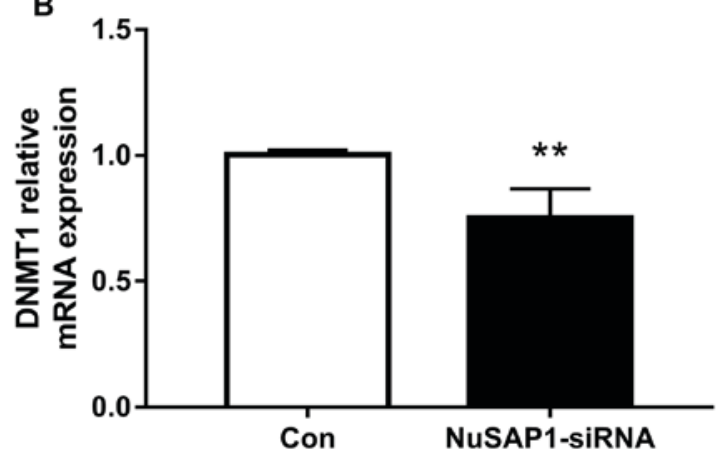

Figure 6. NuSAP1 silencing inhibits the DNMT1 but not GLI1 gene expression in liver cancer cell. (A) The relative GLI1 mRNA level was determined by RT-qPCR inHepG2 cells following treatment with siNuSAP1 for $72 \mathrm{~h}$. $\beta$-actin was used as the internal control. No significant difference in the GLI1 mRNA level was observed following NuSAP1 knockdown. (B) The relative DNMT1 mRNA level was determined by RT-qPCR in HepG2 cells following treatment with siNuSAP1 for $72 \mathrm{~h}$. $\beta$-actin was used as an internal control. NuSAP1 knockdown significantly decreased the mRNA level of DNMT1 ${ }^{* * *} \mathrm{P}<0.01$ vs. the control. NuSAP1, nucleolar and spindle-associated protein 1; DNMT1, DNA methyltransferase; GLI1, glioma-associated oncogene; RT-qPCR, reverse transcription quantitative polymerase chain reaction; si, small interfering RNA.

However, NuSAP1 silencing inhibited the DNMT1 gene expression in liver cancer cells.

In conclusion, the current study demonstrated that NuSAP1 upregulation may be an early event in the processes of liver cancer, therefore NuSAP1 may serve an important role in liver cancer development. NuSAP1 may provide a new target for liver cancer gene therapy. Although the underlying mechanisms require further investigation, the current study provides an improved understanding of the function of NuSAP1 in liver cancer and lays the foundation for the development of novel liver cancer treatment strategies.

\section{Acknowledgements}

Not applicable.

\section{Funding}

The present study was funded by grants from the Health Bureau of Nantong City (grant nos. WKZL2018063, WKZL2018057 and WQ2016011), the Nantong Science and Technology Bureau (grant nos. MS22015105 and GJZ16007), the Natural Science Foundation of Jiangsu Province (grant no. BK20160420) and the National Science Foundation for Young Scientists of China (grant no. 81601842).

\section{Availability of data and materials}

The datasets and databases used and/or analyzed during the current study are available from the corresponding author on reasonable request.

\section{Authors' contributions}

$\mathrm{ZB}$ and $\mathrm{ZL}$ contributed to the study design and literature search. YW contributed to the western blotting, immunohistochemistry, RT-qCPR, writing of the manuscript, statistical analysis and figure creation. FX contributed to the tissue collection and histopathological analysis. HL and LJ performed the flow cytometry. XL and LC contributed to the data collection.

\section{Ethical approval and consent to participate}

The current study was performed in accordance with the ethical guidelines of the 1975 Declaration of Helsinki. The current study was reviewed and approved by the Ethics Committee of the Nantong Third People's Hospital Affiliated to Nantong University.

\section{Patient consent for publication}

Informed consent was obtained from each patient.

\section{Competing interests}

The authors declare that they have no competing interests.

\section{References}

1. Villanueva A and Llovet JM: Liver cancer in 2013: Mutational landscape of HCC-the end of the beginning. Nat Rev Clin Oncol 11: 73-74, 2014.

2. El-Serag HB: Epidemiology of viral hepatitis and hepatocellular carcinoma. Gastroenterology 142: 1264-1273 e1261, 2012.

3. Tsuchiya N, Sawada Y, Endo I, Saito K, Uemura Y and Nakatsura T: Biomarkers for the early diagnosis of hepatocellular carcinoma. World J Gastroenterol 21: 10573-10583, 2015.

4. Raemaekers T, Ribbeck K, Beaudouin J, Annaert W, Van Camp M, Stockmans I, Smets N, Bouillon R, Ellenberg J and Carmeliet G: NuSAP, a novel microtubule-associated protein involved in mitotic spindle organization. J Cell Biol 162: 1017-1029, 2003

5. Verbakel W, Carmeliet G and Engelborghs Y: SAP-like domain in nucleolar spindle associated protein mediates mitotic chromosome loading as well as interphase chromatin interaction. Biochem Biophys Res Commun 411: 732-737, 2011.

6. Ribbeck K, Groen AC, Santarella R, Bohnsack MT, Raemaekers T, Köcher T, Gentzel M, Görlich D, Wilm M, Carmeliet G, et al: NuSAP, a mitotic RanGTP target that stabilizes and cross-links microtubules. Mol Biol Cell 17: 2646-2660, 2006.

7. Li C, Zhang Y, Yang Q, Ye F, Sun SY, Chen ES and Liou YC: NuSAP modulates the dynamics of kinetochore microtubules by attenuating MCAK depolymerisation activity. Sci Rep 6: 18773, 2016.

8. Li C, Xue C, Yang Q, Low BC and Liou YC: NuSAP governs chromosome oscillation by facilitating the Kid-generated polar ejection force. Nat Commun 7: 10597, 2016.

9. Hussain S, Benavente SB, Nascimento E, Dragoni I, Kurowski A, Gillich A, Humphreys P and Frye M: The nucleolar RNA methyltransferase Misu (NSun2) is required for mitotic spindle stability. J Cell Biol 186: 27-40, 2009.

10. Ryu B, Kim DS, Deluca AM and Alani RM: Comprehensive expression profiling of tumor cell lines identifies molecular signatures of melanoma progression. PLoS One 2: e594, 2007. 
11. Bogunovic D, O'Neill DW, Belitskaya-Levy I, Vacic V, Yu YL, Adams S, Darvishian F, Berman R, Shapiro R, Pavlick AC, et al: Immune profile and mitotic index of metastatic melanoma lesions enhance clinical staging in predicting patient survival. Proc Natl Acad Sci USA 106: 20429-20434, 2009.

12. Chen DT, Nasir A, Culhane A, Venkataramu C, Fulp W, Rubio R, Wang T, Agrawal D, McCarthy SM, Gruidl M, et al: Proliferative genes dominate malignancy-risk gene signature in histologically-normal breast tissue. Breast Cancer Res Treat 119: 335-346, 2010.

13. Chen L, Yang L, Qiao F, Hu X, Li S, Yao L, Yang XL and Shao ZM: High levels of nucleolar spindle-associated protein and reduced levels of BRCA1 expression predict poor prognosis in triple-negative breast cancer. PLoS One 10: e0140572, 2015.

14. Marie SK, Okamoto OK, Uno M, Hasegawa AP, Oba-Shinjo SM, Cohen T, Camargo AA, Kosoy A, Carlotti CG Jr, Toledo S, et al: Maternal embryonic leucine zipper kinase transcript abundance correlates with malignancy grade in human astrocytomas. Int J Cancer 122: 807-815, 2008 .

15. Zhang M, Yang D, Liu X, Liu Y, Liang J, He H, Zhong K, Lin L, Tao G, Zhang C and Zhou J: Expression of Nusap1 in the surgical margins of hepatocellular carcinoma and its association with early recurrence. Nan Fang Yi Ke Da Xue Xue Bao 33: 937-938, inside back cover, 2013 (In Chinese).

16. Gulzar ZG, McKenney JK and Brooks JD: Increased expression of NuSAP in recurrent prostate cancer is mediated by E2F1. Oncogene 32: 70-77, 2013.

17. Xue YP, Ji XY, Yang L, Liu HR, Sheng YJ, Dai XX, Xi YJ, Liu JC, Shi J, Xie T, et al: Experimental studies on correlation between nucleolus spindle-related protein 1 and the malignant progression and prognosis of human glioblastoma multiforme. Zhonghua Yi Xue Za Zhi 98: 340-345, 2018 (In Chinese; Abstract available in Chinese from the publisher).

18. Wu X, Xu B, Yang C, Wang W, Zhong D, Zhao Z, He L, Hu Y, Jiang L, Li J, et al: Nucleolar and spindle associated protein 1 promotes the aggressiveness of astrocytoma by activating the Hedgehog signaling pathway. J Exp Clin Cancer Res 36: 127, 2017.

19. Stuart JE, Lusis EA, Scheck AC, Coons SW, Lal A, Perry A and Gutmann DH: Identification of gene markers associated with aggressive meningioma by filtering across multiple sets of gene expression arrays. J Neuropathol Exp Neurol 70: 1-12, 2011.

20. Cui H, Li TL, Guo HF, Wang JL, Xue P, Zhang Y, Fan JH, Li ZP and Gao YJ: Silymarin-mediated regulation of the cell cycle and DNA damage response exerts antitumor activity in human hepatocellular carcinoma. Oncol Lett 15: 885-892, 2018.

21. Mills CA, Suzuki A, Arceci A, Mo JY, Duncan A, Salmon ED and Emanuele MJ: Nucleolar and spindle-associated protein 1 (NUSAP1) interacts with a SUMO E3 ligase complex during chromosome segregation. J Biol Chem 292: 17178-17189, 2017.

22. Vogel A, Cervantes A, Chau I, Daniele B, Llovet J, Meyer T, Nault JC, Neumann U, Ricke J, Sangro B, et al: Hepatocellular carcinoma: ESMO Clinical Practice Guidelines for diagnosis, treatment and follow-up. Ann Oncol 29: iv238-iv255, 2018.

23. Ju LL, Chen L, Li JH, Wang YF, Lu RJ, Bian ZL and Shao JG: Effect of NDC80 in human hepatocellular carcinoma. World J Gastroenterol 23: 3675-3683, 2017.

24. Rhodes DR, Kalyana-Sundaram S, Mahavisno V, Varambally R, Yu J, Briggs BB, Barrette TR, Anstet MJ, Kincead-Beal C, Kulkarni P, et al: Oncomine 3.0: Genes, pathways, and networks in a collection of 18,000 cancer gene expression profiles. Neoplasia 9: 166-180, 2007.
25. Chandrashekar DS, Bashel B, Balasubramanya SAH, Creighton CJ, Ponce-Rodriguez I, Chakravarthi BVSK and Varambally S: UALCAN: A portal for facilitating tumor subgroup gene expression and survival analyses. Neoplasia 19: 649-658, 2017.

26. Lánczky A, Nagy Á, Bottai G, Munkácsy G, Szabó A, Santarpia L and Győrffy B: miRpower: A web-tool to validate survival-associated miRNAs utilizing expression data from 2178 breast cancer patients. Breast Cancer Res Treat 160: 439-446, 2016

27. Anaya J: OncoLnc: Linking TCGA survival data to mRNAs, miRNAs, and lncRNAs. PeerJ Computer Science 2: e67, 2016.

28. Livak KJ and Schmittgen TD: Analysis of relative gene expression data using real-time quantitative PCR and the 2 (-Delta Delta C (T)) method. Methods 25: 402-408, 2001.

29. Peterson KA, Nishi Y, Ma W, Vedenko A, Shokri L, Zhang X, McFarlane M, Baizabal JM, Junker JP, van Oudenaarden A, et al: Neural-specific Sox2 input and differential Gli-binding affinity provide context and positional information in Shh-directed neural patterning. Genes Dev 26: 2802-2816, 2012.

30. Hui CC and Angers S: Gli proteins in development and disease. Annu Rev Cell Dev Biol 27: 513-537, 2011.

31. Kotian S, Banerjee T, Lockhart A, Huang K, Catalyurek UV and Parvin JD: NUSAP1 influences the DNA damage response by controlling BRCA1 protein levels. Cancer Biol Ther 15: 533-543, 2014.

32. Shukla V, Coumoul X, Lahusen T, Wang RH, Xu X, Vassilopoulos A, Xiao C, Lee MH, Man YG, Ouchi M, et al: BRCA1 affects global DNA methylation through regulation of DNMT1. Cell Res 20: 1201-1215, 2010.

33. Iyer J, Moghe S, Furukawa M and Tsai MY: What's Nu (SAP) in mitosis and cancer? Cell Signal 23: 991-998, 2011.

34. Vanden Bosch A, Raemaekers T, Denayer S, Torrekens S, Smets N, Moermans K, Dewerchin M, Carmeliet P and Carmeliet G: NuSAP is essential for chromatin-induced spindle formation during early embryogenesis. J Cell Sci 123: 3244-3255, 2010.

35. Yu HT and Lu PR: Effects of lentiviral short hairpin RNA silencing of Toll-like receptor 4 on the lens epithelial cell line HLEC. Genet Mol Res 15: gmr7897, 2016.

36. Zhang L, Liu HJ, Li TJ, Yang Y, Guo XL, Wu MC, Rui YC and Wei LX: Lentiviral vector-mediated siRNA knockdown of SR-PSOX inhibits foam cell formation in vitro. Acta Pharmacol Sin 29: 847-852, 2008

37. Hanahan D and Weinberg RA: Hallmarks of cancer: The next generation. Cell 144: 646-674, 2011.

38. Lauss M, Kriegner A, Vierlinger K, Visne I, Yildiz A, Dilaveroglu E and Noehammer C: Consensus genes of the literature to predict breast cancer recurrence. Breast Cancer Res Treat 110: 235-244, 2008.

39. Gordon CA, Gong X, Ganesh D and Brooks JD: NUSAP1 promotes invasion and metastasis of prostate cancer. Oncotarget 8: 29935-29950, 2017.

(i) $(5)$ This work is licensed under a Creative Commons Attribution-NonCommercial-NoDerivatives 4.0 International (CC BY-NC-ND 4.0) License. 